\title{
The Comparative Study Of Efficiency Of Intravenous Heparin And Oral Aspirin On 48 Hours Beginning Primary Medication In Ischemic Stroke Patients
}

\author{
Seyed Ehsan Asadi \\ MSc in Nursing, Esfahan Medical University, Esfahan, Iran \\ E-mail: ehsanasadi26@yahoo.com
}

Objective: This study was done for comparing efficiency of Intravenous Heparin and Oral Aspirin among patients who had cerebral thrombi embolitic vascular accidents. Effectives of Heparin and Aspirin on prognosis and recovery of these patients were evaluated.

Method: This study was a clinical randomized trial. Patients were classified randomly in one of the heparin ( $n=40)$ and one group Aspirin $(n=40)$ treatment. At first in the Heparin treatment group, patients were medicated by Heparin $100 \mathrm{IU} / \mathrm{kg}$ and then it was followed by $1000 \mathrm{IU} /$ hour for 48 hours. In the Aspirin treated group, whom took $325 \mathrm{mg} /$ day for 48 hours. The effectiveness of both treatments were evaluated after 48 hours on neuro-muscular, speech, vision, and sphincter function.

Results: The results showed that both treatments were effective on improvement of neuro-muscular, speech, vision, and sphincter function, while Heparin effectiveness was more than Aspirin. Improvement of Nervous function in the Heparin group was $53.2 \%$ in compare of $31.2 \%$ for Aspirin $(p=0.40)$. In addition, in the Heparin treatment group $59.6 \%$ of muscular dysfunction was attained optimum muscular function in compare of $30.7 \%$ with Aspirin $(p=0.001)$. It was found significant relation for improvement of speech function in Heparin treatment group $(\mathrm{P}=0.01)$. There were not a significant finding relation for improvement of vision, and sphincter function between Heparin and Aspirin treated groups.

Conclusion: Beginning primary medication with Heparin would be effective for achieving optimum function among patients with cerebral thrombi embolitic vascular accidents.

Key words: Aspirin, Heparin, Stroke, Ischemy 